\title{
Role of anxiety in young children's pain memory development after surgery
}

Shanaya Fischer ${ }^{a}$, Jillian Vinall ${ }^{b}$, Maria Pavlova ${ }^{a}$, Susan Graham ${ }^{a, c}$, Abbie Jordan ${ }^{d}$, Jill Chorney ${ }^{e}$, Nivez Rasic ${ }^{b}$, James T. Brookes ${ }^{f, g}$, Monica Hoy ${ }^{f}$, Warren K. Yunker ${ }^{f, g}$, Melanie Noel ${ }^{h, *}$

\begin{abstract}
Pediatric pain is common, and memory for it may be distressing and have long-lasting effects. Children who develop more negatively biased memories for pain (ie, recalled pain is higher than initial pain report) are at risk of worse future pain outcomes. In adolescent samples, higher child and parent catastrophic thinking about pain was associated with negatively biased memories for postsurgical pain. This study examined the influence of child and parent anxiety on the development of younger children's postsurgical pain memories. Seventy-eight children undergoing a tonsillectomy and one of their parents participated. Parents reported on their anxiety (state and trait) before surgery, and trained researchers observationally coded children's anxiety at anaesthesia induction. Children reported on their postsurgical pain intensity and pain-related fear for 3 days after discharge. One month after surgery, children recalled their pain intensity and pain-related fear using the same scales previously administered. Results revealed that higher levels of postsurgical pain and higher parent trait anxiety predicted more negatively biased memories for pain-related fear. Parent state anxiety and child preoperative anxiety were not associated with children's recall. Children who developed negatively biased pain memories had worse postsurgical pain several days after surgery. These findings underscore the importance of reducing parental anxiety and effective postsurgical pain management to potentially buffer against the development of negatively biased pain memories in young children.
\end{abstract}

Keywords: Postsurgical pain, Acute pain, Parents, Children, Anxiety, Memory

\section{Introduction}

Children's memories for pain are a robust predictor of future pain experiences. $^{29}$ These memories are highly susceptible to distortion. Children can either recall pain accurately (ie, recall the same level of pain as initially reported) or in a positively or negatively biased (ie, recall less or more pain than initially reported) way. ${ }^{29}$ Negatively biased pain memories are linked to greater future pain intensity, ${ }^{27}$ fear, ${ }^{27}$ and distress. ${ }^{27}$ Moreover, amongst adolescents undergoing major surgery, more negatively biased memories for pain predicted higher postsurgical pain at a time when pain can transition to a chronic state. ${ }^{33}$ As such, it is critical to identify risk factors for pain memory biases, particularly early in development before pain problems first emerge.

Sponsorships or competing interests that may be relevant to content are disclosed at the end of this article.

Departments of a Psychology and, ${ }^{b}$ Anesthesia, University of Calgary, Calgary, AB, Canada, ${ }^{C}$ Owerko Centre, Alberta Children's Hospital Research Institute, University of Calgary, Calgary, AB, Canada, ${ }^{d}$ Department of Psychology and Centre for Pain Research, University of Bath, Bath, United Kingdom, ${ }^{e}$ Department of Anesthesia, Pain Management and Perioperative Medicine, Dalhousie University, Halifax, NS, Canada, ${ }^{f}$ Section of Otolaryngology_-Head and Neck Surgery, Department of Surgery, Cumming School of Medicine, University of Calgary, Calgary, AB, Canada, ${ }^{g}$ Section of Pediatric Surgery, Department of Surgery, Cumming School of Medicine, University of Calgary, Calgary, AB, Canada, ${ }^{h}$ Department of Psychology, University of Calgary and Alberta Children's Hospital Research Institute, Calgary, $A B$, Canada

${ }^{*}$ Corresponding author. Address: Department of Psychology, University of Calgary, 2500 University Dr NW, Calgary, AB, T2N 1N4, Canada. Tel.:+1 (403) 220-4969. E-mail address: melanie.noel@ucalgary.ca (M. Noel).

PAIN 160 (2019) 965-972

(C) 2018 International Association for the Study of Pain

http://dx.doi.org/10.1097/j.pain.0000000000001473
Theoretical and empirical research has implicated anxiety in the development of negatively biased memories, ${ }^{3}$ including memories for pain. ${ }^{29,32}$ Highly anxious children may selectively attend to, encode, and subsequently recall threatening and painful information. ${ }^{1}$ Elevated levels of anxiety are associated with higher levels of postsurgical pain intensity and distress, ${ }^{7}$ which is a strong driver of negative biases in pain recall. ${ }^{28,34}$ Moreover, among children undergoing acute experimental (cold pressor), needle pain, and dental pain, higher state and trait anxiety were related to more negatively biased memories for pain. $21,28,37,43$

Developmental models of children's pain memory development posit that parents are also critically important in influencing children's explicit memories for pain. ${ }^{29,31}$ This is believed to be particularly true during the period of early childhood when children's memories are most susceptible to distortion due to suggestibility effects. ${ }^{2}$ Through language-based interactions between parents and children, highly anxious parents may repeatedly emphasize threatening details of the child's pain experience, leading to heightened attentional processing of threat and subsequent distortions in memory that prioritize those threatening details. Research has demonstrated that adolescents whose parents tended to ruminate more about, and magnify the threat value of, their adolescent's pain before surgery, subsequently, developed more negatively biased pain memories. ${ }^{34}$ Moreover, these negatively biased pain memories placed children at higher risk of poorer postsurgical pain outcomes. ${ }^{33}$

Pain memories are believed to be most malleable and influenced by parents during early childhood ${ }^{31}$; however, research has not examined anxiety-related risk factors for biases in young children's pain memory development after surgery. To address this critical gap, this study examined the influence of parent and child anxiety on the development of young children's 
memories for pain after a surgical procedure (tonsillectomy) and the relation between negatively biased pain memories and postsurgical pain and fear during the recovery phase (ie, the first few days at home after surgery). Consistent with conceptual models of children's acute pain memory development, ${ }^{29}$ we hypothesized that (1) greater parent (state and trait) anxiety and (2) greater child preoperative anxiety would be associated with negatively biased memories for pain intensity and pain-related fear. We also hypothesized that children who developed negatively biased pain memories would have higher levels of postsurgical pain and fear during the recovery phase.

\section{Methods}

\subsection{Participants and setting}

Eighty-six children and one of their parents participated in this study. Parent-child dyads were recruited from a Pediatric Otolaryngology Clinic at a tertiary-level children's hospital in Western Canada. Children were eligible if they were between the ages of 5 and 7 years, were scheduled to undergo a tonsillectomy (with or without adenoidectomy), were able to speak and understand English, and were American Society of Anesthesiology physical status I or II. Children, who had psychiatric or developmental disorders (as reported by parents during the recruitment call), were receiving premedication anxiolytics (eg, midazolam), or had a health condition that interfered with the standard anaesthesia protocol (eg, congenital heart disease; ie, ASA physical status III or higher), were excluded from the study.

Eighty-six parent-child dyads were enrolled in the study, 3 dropped out, and 5 could not be reached for follow-up. Thus, the final sample included 78 parent-child dyads ( $41 \%$ girls, $M_{\text {age }}=$ 5.82 [SD $=0.80$ ], 70.5\% mothers). The majority of participants were white $(82.1 \%)$ and reported an average annual household income of $\$ 70,000$ or greater (76.3\%). Key demographic variables (annual household income, age, sex, and ethnicity) did not differ between those dyads who did and did not complete the study $(P S>0.05)$. Sociodemographic characteristics of the sample are summarized in Table 1. The institutional health research ethics board approved the study.

\subsection{Measures}

\subsubsection{Parent measures}

\subsubsection{Sociodemographics}

Parents reported on their annual household income, sex, and ethnicity, and their child's sex, age, and ethnicity.

\subsubsection{State and trait anxiety}

Parent state and trait anxiety were assessed using the State-Trait Anxiety Inventory (STAl). ${ }^{41}$ The STAl consists of 2 subscales: State (STAI-S) and Trait (STAI-T). Twenty items of each subscale are rated on a 4-point Likert scale (1 = "almost never," 2 = "sometimes," 3 = "often," and 4 = "almost always"). Higher scores indicate greater levels of state and trait anxiety. The STAI-S items refer to how an individual is feeling in a moment (eg, "I am tense"), whereas the STAI$\mathrm{T}$ items refer to how an individual generally feels (eg, "I worry too much over something that really doesn't matter"). The STAI has been used in pediatric surgery research ${ }^{19}$ and has good test-retest reliability $(r=0.31-0.86)^{17}$ and good construct validity. ${ }^{17}$ Internal consistency in the present sample was $\alpha=0.90$ and $\alpha=0.87$ for state and trait subscales, respectively.
Table 1

\section{Characteristics of the sample.}

\begin{tabular}{lll}
\hline & Mean & \\
\hline Child age (y) & 5.82 & SD \\
\hline & & 0.80 \\
\hline Child sex (female) & Frequency (\%) \\
\hline Parent sex (female) & 41.0 \\
\hline Parent ethnicity & 70.5 \\
Aboriginal & \\
Arab/West Asian & 3.8 \\
Filipino & 1.3 \\
South Asian & 5.1 \\
South East Asian & 2.6 \\
White (Caucasian) & 1.3 \\
Other & 82.1 \\
\hline
\end{tabular}

Annual household income

$<\$ 10,000$

$\$ 10,000-\$ 29,000$

$\$ 30,000-\$ 49,000$

$\$ 50,000-\$ 69,000$

$>\$ 70,000$

Did not respond

\section{3}

5.2

5.2

11.8

76.3

2.6

Surgical procedure

Tonsillectomy

Tonsillectomy and adenoidectomy ( $T$ and $A$ )

6.4

$T$ and $A$ and nose sinoscopy

$T$ and $A$ and ear myringotomy

67.9

23.1

6.4

\subsubsection{Child measures}

\subsubsection{Preoperative anxiety}

Anxiety is a negative emotional state characterized by anticipation of future threat. ${ }^{25}$ To capture this construct behaviourally in young children, the Modified Yale Preoperative Anxiety Scale (mYPAS) ${ }^{20}$ was used. The mYPAS is an 18-item observational tool that assesses 4 domains of preoperative child behaviour (ie, activity, vocalization, emotional expressivity, and state of arousal) in the holding area and the operating room. Higher scores on this scale indicate higher levels of anxiety. The mYPAS has demonstrated good intraobserver reliability $(\kappa=0.66-0.91)$ and construct validity when compared with other measures of anxiety $(r=0.59$ $0.64) .{ }^{20}$ In line with the previous studies, ${ }^{12,23}$ preoperative anxiety was assessed during inhalational anaesthesia induction because this is when preoperative anxiety levels peak. ${ }^{12,18}$ For this study, one of the authors (J.V.) completed an intensive, in-person training on the mYPAS scale and subsequently trained 2 onsite reliability coders (M.P. and S.F.). Reliability coders each coded a minimum of 10 surgeries until they reached reliability of at least $\alpha$ $=0.75$ on each domain. Twenty-two cases (26.50\%) were double-coded live for interrater reliability. Interrater reliability scores were $\alpha=0.91, \alpha=0.98, \alpha=0.95$, and $\alpha=0.93$ for activity, vocalization, emotional expressivity, and state of arousal, respectively. Internal consistency was high ( $\alpha=0.92)$.

\subsubsection{Pain-related fear}

In contrast to anxiety, fear is a proximal alarm reaction to an immediate threat. ${ }^{25}$ Both anxiety and fear are important to assess in the context of pain. ${ }^{25}$ Similar to previous research, children's pain-related fear and recall of pain-related fear were assessed using the single-item Children's Fear Scale (CFS). ${ }^{24}$ Children 
rated their pain-related fear using 5 faces that depict varying degrees of fear with anchors "not at all scared" (0) to "most scared possible" (4). ${ }^{24}$ The CFS has demonstrated good test-retest reliability $(r=0.76)$ and construct validity $(r=0.73) .{ }^{24}$ The CFS has been used previously to assess recall of pain-related fear in children aged 5 to 10 years. ${ }^{30}$

\subsubsection{Pain intensity}

Children's postsurgical pain intensity and recall of postsurgical pain were assessed using the single-item Faces Pain ScaleRevised (FPS-R) ${ }^{15}$ Children rated their pain intensity using 6 sexneutral faces that vary in pain expression from neutral $(0)$ to extreme (10) pain facial expressions. The FPS-R is a recommended self-report measure of pain intensity in children of this age range and has demonstrated good test-retest reliability $(r=0.79)$ and construct validity $(r=0.59-0.90) .{ }^{42}$ The FPS-R has been used previously to assess recall of pain intensity in children aged 5 to 10 years. ${ }^{30}$

\subsubsection{Memory interview}

In line with previous research with children of this age range, ${ }^{30}$ a trained research assistant called the children on the telephone 1 month after surgery to assess their recall of pain intensity and pain-related fear using the FPS-R and the CFS. Parents were provided with hard copies of the pain intensity and pain-related fear scales on the day of surgery, so that pain could be subsequently assessed during the postoperative period and the memory interview. Randomly ordered letters were placed under each face on the scales. Numbers were not used to avoid a potential confounding effect. ${ }^{30}$ Parents were asked to orient the child to look at the correct scale, but they were explicitly instructed to refrain from influencing their child's response/ rating. During the interview, the research assistant provided instructions for each scale and oriented children to the letters below the faces. Prompted by the researcher, children then rated their recalled pain intensity (FPS-R) and pain-related fear (CFS) on the 1st day and on the first few days after the surgery. We used this approach to be consistent with the established memory interview protocol that has been used in pediatric samples undergoing surgery, ${ }^{34}$ which elicits children's memory of pain intensity and pain-related fear on day 1 and the average days 1 to 3 after surgery. Both pain intensity and pain-related fear were assessed to capture the multidimensional nature of children's pain and pain memories. ${ }^{35}$ Telephone interviews have been previously used to assess children's memories for pain in children aged 5 to 10 years. $^{30}$

\subsection{Procedure}

Before their surgery, a member of the Pediatric Otolaryngology Clinic informed parents of children between the ages of 5 and 7 about the study and provided interested parents a permission to contact form. One to 3 weeks before surgery, interested parents were contacted by the study team through telephone. Parents of eligible children were sent an online consent form and questionnaires (sociodemographics and STAI-T) through REDCap, a secure online survey system. ${ }^{14}$ On the day of surgery, parents rated their state anxiety (STAI-S), and a research assistant coded child preoperative anxiety at anesthesia induction (mYPAS). A standardized anesthetic protocol was used for each child. Anesthesia induction was achieved via inhalation of sevoflurane $/ \mathrm{O}_{2} / \mathrm{N}_{2} \mathrm{O}$ /through a mask. An intravenous catheter was then inserted, and anesthesia was maintained using either volatile anesthetic or total intravenous anesthetic for the procedure. Patients were maintained using either sevoflurane or propofol with or without the remifentanil titrated to effect to provide a balanced anesthetic. During the procedure, all patients received dexamethasone $0.2 \mathrm{mg} / \mathrm{kg}$ intravenously (IV), ondansetron $0.1 \mathrm{mg} / \mathrm{kg} \mathrm{IV}$, and morphine for analgesia. The majority of children (74\%) were discharged on the same day and did not differ from children admitted for an overnight stay (26\%) on any of the key variables ( $P S>0.05)$.

As has been performed in the previous research, ${ }^{22}$ after their child's surgery, parents received the faces scales (FPS-R and CFS) for at home assessment of their child's pain intensity and pain-related fear as well as for the memory interview. For the athome assessment, parents were provided with written and verbal instructions on how to administer the FPS-R and the CFS to their child at home on days 1, 2, and 3 after surgery (day 1: 1st day after surgery). On each postsurgical day, parents were emailed a REDCap survey at 5:00 PM prompting them to record their child's report of pain intensity and pain-related fear for that day. The majority of parents (64\%) completed the surveys on the day they were sent. There were no differences on any of the key variables between parents who completed the surveys on the day they were sent or on a different day $(P S>0.05)$. Approximately one month after surgery $(\mathrm{M}=31.49$ days, $\mathrm{SD}=4.63$ days, range $=25-50$ days), a research assistant conducted the telephone memory interview with the child. ${ }^{30}$ Pain recall was not affected by the timing of the memory interviews ( $P S>0.05)$. Children and parents received a $\$ 40$ gift card upon completion of the study.

\section{Statistical analyses}

Statistical analyses were conducted using the Statistical Package for Social Sciences (SPSS), Version 24.0. Descriptive, correlational (bivariate and partial), and hierarchical regression analyses were conducted using 2-tailed hypothesis testing. Similar to previous research, ${ }^{34}$ child report of postsurgical pain intensity and pain-related fear was averaged over days 1,2, and 3 to correspond with the memory interview questions (ie, children's recall of pain intensity and pain-related fear during the first few days after surgery). In cases where there were missing data for one of the days (13\%), a 2-day average was taken. Bivariate correlations were conducted to examine the relations between age and key variables. $T$ tests were conducted to examine the association of child sex with key variables. A multivariate analysis of variance was conducted to examine the association between annual household income and key variables. Preliminary analyses revealed that there were no significant associations between child age, sex, or household income, and any of the key variables ( $P$ s $>0.05)$.

Similar to previous research, to examine the influence of pain memory biases on postsurgical outcomes (ie, levels of pain intensity and pain-related fear on days 1, 2, and 3 after surgery), children were categorized into 2 groups based on their recall of pain intensity and pain-related fear at 2 time points (ie, day 1 after surgery and the first 1-3 days after surgery). ${ }^{27,30}$ "Negative estimators" recalled higher levels of pain or pain-related fear as compared to their initial reports (ie, the difference between the recall and initial ratings was $\geq 1$ face on the FPS-R or the CFS). "Accurate/positive estimators" recalled the same or lower levels of pain or pain-related fear as compared to their initial reports (ie, the difference between the recall and initial ratings was $\leq 1$ face or $=0$ on the FPS-R or the CFS). For example, if a child rated their level of pain intensity as 5/10 on day 1 after surgery, but subsequently recalled their level of pain intensity as 10/10, they 
were categorized as a "negative estimator (pain intensity on day 1)." Conversely, if the child recalled their level of pain intensity as $\leq 5 / 10$, they were categorized as an "accurate/positive estimator (pain intensity on day 1)." Overall, 8 groups were created: negative and accurate/positive estimators (pain intensity on day 1); negative and accurate/positive estimators (pain-related fear on day 1); negative and accurate/positive estimators (average pain intensity on days 1-3); and negative and accurate/positive estimators (average pain-related fear on days 1-3). The groups' ratings of postsurgical pain and pain-related fear were compared using independent-samples $t$ tests.

In line with previous research with older children, $28,30,34$ hierarchical regression analyses were used to test parent and child anxiety as predictors of children's memory biases for pain intensity and pain-related fear. From previous research, variables were entered in the models as follows: initial pain ratings (corresponding to each respective memory question) were controlled for in the first step $^{28,30,34}$; child preoperative anxiety was entered in the second step; and parent anxiety was entered in the third step to examine the influence of parent anxiety on children's pain memories above and beyond child anxiety and initial pain ratings. ${ }^{34}$ Hierarchical regression analyses included 75 participants. Three participants were removed because of missing data on the observational measure of child anxiety at anxiety induction (mYPAS). Participants who had missing data did not significantly differ from those with complete data on any key variables ( $P s>0.05)$.

\section{Results}

\subsection{Descriptive statistics}

Descriptive statistics of the key variables are summarized in Table 2. Children reported a mean pain intensity of 4.08/10 (SD = $3.10)$ on day 1 after surgery and of $3.83 / 10(S D=2.17)$ for the average of the first few days after surgery. Children's recalled mean pain intensity levels for day 1 after surgery and the first few days after surgery were 4.79/10 (SD = 3.75) and 4.31/10 (SD = 3.30), respectively. Children reported mean pain-related fear ratings of $0.74 / 4(S D=1.15)$ on day 1 after surgery and of $0.72 / 4$ $(S D=0.91)$ for the average of the first few days after surgery. Children's recalled mean pain-related fear levels for day 1 after surgery and the first few days after surgery were 1.24/4 (SD = 1.33) and 1.21/4 (SD = 1.31), respectively.

\subsection{Bivariate correlations}

Bivariate correlations are summarized in Table 3. Postsurgical pain intensity on day 1 was positively correlated with recall of pain intensity on day $1(r=0.33, P<0.01)$. Postsurgical pain intensity averaged over the first few days after surgery was positively correlated with recall of pain intensity and pain-related fear on the first few days after surgery $(r=0.29, P<0.05, r=0.33, P<0.01$, respectively). Pain-related fear averaged over the first few days after surgery was positively correlated with recall of pain-related fear on day 1 ( $r=0.33, P<0.01)$, and with recall of pain intensity $(r=0.31, P<0.01)$ and recall of pain-related fear $(r=0.43, P<$ 0.001 ) on the first few days after surgery.

\subsection{Hierarchical regression analyses}

The results of the hierarchical regression analyses are summarized in Table 4. Four hierarchical regression models were conducted to examine (1) children's memory for pain intensity on day 1 after surgery, (2) children's memory for pain-related fear on day 1 after surgery, (3) children's memory for pain intensity on the first few days after surgery, and (4) children's memory for painrelated fear on the first few days after surgery.

\subsubsection{Model 1. Children's memories for pain intensity (day 1)}

Children's initial level of experienced pain intensity on day 1 after surgery accounted for a significant proportion of variance $(11 \%)$ in children's memories for pain intensity on day $1(\Delta \mathrm{F}(1,73)=9.00, P$ $<0.01)$. After controlling for initial ratings of pain intensity, and child preoperative anxiety, parent state and trait anxiety accounted for $4 \%$ of variance in the model $(\Delta \mathrm{F}(2,70)=1.80, P>0.05)$.

\subsubsection{Model 2. Children's memories for pain-related fear (day 1)}

Children's initial rating of pain-related fear on day 1 after surgery accounted for $5 \%$ of variance in children's memories for pain-related fear on day $1(\Delta \mathrm{F}(1,73)=3.64, P>0.05)$. Similarly, parent state and trait anxiety did not account for a significant amount of variance, above and beyond initial ratings of pain-related fear and child preoperative anxiety $\left(\Delta \mathrm{R}^{2}=0.02, \Delta \mathrm{F}(2,70)=0.90, P>0.05\right)$.

\subsubsection{Model 3. Children's memories for pain intensity (first few days)}

Children's initial ratings of pain intensity during the first few days after surgery (ie, the average pain intensity reported over days 1-3 after surgery) accounted for a significant proportion of variance $(9 \% ; \Delta \mathrm{F}(1,73)=7.14, P<0.01)$. Parent state and trait anxiety did not account for a significant proportion of variance in children's memories for pain intensity on the first few days home after surgery, above and beyond initial pain intensity ratings and child preoperative anxiety $\left(\Delta \mathrm{R}^{2}=0.01, \Delta \mathrm{F}(2,70)=0.45, P>0.05\right)$.

Table 2

Descriptive data for key variables $(\mathrm{N}=78)$.

\begin{tabular}{llllll}
\hline Variable & Mean & SD & Variable & Mean & SD \\
\hline Pain intensity (day 1) & 4.08 & 3.10 & Memory for pain intensity (day 1) & 4.79 & 3.75 \\
\hline Pain-related fear (day 1) & 0.74 & 1.15 & Memory for pain-related fear (day 1) & 1.24 & 1.33 \\
\hline Pain intensity (days 1-3) & 3.83 & 2.17 & Memory for pain intensity (days 1-3) & 4.31 & 1.21 \\
\hline Pain-related fear (days 1-3) & 0.72 & 0.91 & Memory for pain-related fear (days 1-3) & 1.31 \\
\hline Child preoperative anxiety & 42.56 & 21.80 & & & \\
\hline Parent state anxiety & 34.45 & 8.68 & & \\
\hline Parent trait anxiety & 35.17 & 7.01 & & \\
\hline
\end{tabular}


Table 3

Correlations of key variables $(\mathrm{N}=78)$.

\begin{tabular}{|c|c|c|c|c|c|c|c|c|c|c|c|}
\hline Variable & 1 & 2 & 3 & 4 & 5 & 6 & 7 & 8 & 9 & 10 & 11 \\
\hline 1. Preoperative anxiety (mYPAS) & - & & & & & & & & & & \\
\hline 2. Parent state anxiety & -0.13 & - & & & & & & & & & \\
\hline 3. Parent trait anxiety & 0.15 & 0.05 & - & & & & & & & & \\
\hline 4. Pain intensity (Day 1) & -0.02 & 0.04 & 0.02 & - & & & & & & & \\
\hline 5. Pain-related fear (day 1) & 0.09 & -0.12 & 0.03 & $0.52^{*}$ & - & & & & & & \\
\hline 6. Pain intensity (days 1-3) & -0.004 & 0.04 & 0.10 & $0.73^{*}$ & $0.47^{\star}$ & - & & & & & \\
\hline 7. Pain-related fear (days 1-3) & 0.01 & -0.003 & -0.02 & $0.41^{*}$ & $0.75^{\star}$ & $0.64^{*}$ & - & & & & \\
\hline 8. Memory for pain intensity (day 1) & -0.13 & 0.20 & 0.01 & $0.33 \dagger$ & 0.10 & $0.36 \dagger$ & 0.11 & - & & & \\
\hline 9. Memory for pain-related fear (day 1) & -0.06 & 0.11 & 0.10 & 0.05 & 0.20 & 0.22 & $0.33+$ & 0.16 & - & & \\
\hline 10. Memory for pain-intensity (days 1-3) & -0.07 & 0.12 & 0.05 & 0.13 & 0.21 & $0.29 \ddagger$ & $0.31 \dagger$ & $0.45^{\star}$ & $0.47^{\star}$ & - & \\
\hline 11. Memory for pain-related fear (days 1-3) & -0.05 & 0.13 & 0.22 & 0.11 & $0.31 \dagger$ & $0.33 \dagger$ & $0.43^{*}$ & 0.08 & $0.56^{\star}$ & $0.49^{*}$ & - \\
\hline
\end{tabular}

${ }^{*} P<0.001$

$\dagger P<0.01$.

$\ddagger P<0.05$.

mYPAS, Modified Yale Preoperative Anxiety Scale.

\subsubsection{Model 4. Children's memories for pain-related fear (first few days)}

Children's initial ratings of pain-related fear were a significant predictor of children's memories for pain-related fear during the first few days after surgery, accounting for $21 \%$ of the variance $(\Delta F(1,73)$ $=19.86, P<0.001)$. After controlling for initial ratings of pain-related fear and child preoperative anxiety, parent trait anxiety, but not parent state anxiety or child preoperative anxiety, significantly accounted for an additional $7 \%$ of variance $(\Delta \mathrm{F}(2,70)=3.57, P<$ $0.05)$. A change in parent trait anxiety from the lowest score (ie, 20) to the highest score (ie, 80 ) is associated with a change in 1.2 faces on the CFS, a clinically significant change in memory for fear of pain.

\subsection{Differences between memory style subgroups on postsurgical pain and fear}

"Negative estimators (pain intensity on day 1 )" reported significantly lower pain intensity $(\mathrm{M}=3.11 / 10, \mathrm{SD}=2.11)$ on day 1 after surgery vs "accurate/positive estimators (pain intensity on day 1)" $(\mathrm{M}=4.90 / 10, \mathrm{SD}=3.57), \mathrm{F}(1,76)=7.00, P<0.05$. "Negative estimators (pain-related fear on day 1 )" reported significantly lower pain intensity $(M=3.19 / 10, S D=2.63)$ and pain-related fear $(M=$ $0.34 / 4, S D=0.83$ ) on day 1 after surgery vs "accurate/positive estimators (pain-related fear on day 1 )" (pain intensity $M=4.70 / 10$, $\mathrm{SD}=3.27$; pain-related fear $\mathrm{M}=1.02 / 4, \mathrm{SD}=1.26), \mathrm{F}(1,76)=$ $4.68, F(1,76)=7.15$, respectively, $P s<0.05$. However, "negative estimators (pain-related fear on day 1 )" reported significantly higher pain intensity $(M=4.31 / 10, S D=2.88)$ on day 3 after surgery vs "accurate/positive estimators (pain-related fear on day 1$)$ " ( $\mathrm{M}=$ 2.74/10, SD = 2.83), $\mathrm{F}(1,73)=5.56, P<0.05$.

\section{Discussion}

This study examined the influence of parent and child anxiety on young children's recall of postsurgical pain, as well as the relations between negatively biased pain memories and

Table 4

Hierarchical regression analyses of children's memories for pain intensity and pain-related fear $(N=75)$.

\begin{tabular}{|c|c|c|c|c|c|}
\hline Criterion variable & Step & Predictor & Beta & $\Delta \mathrm{R}^{2}$ & Cumulative $\mathrm{R}^{2}$ \\
\hline Memory for pain intensity (day 1) & $\begin{array}{l}1 \\
2 \\
3\end{array}$ & $\begin{array}{l}\text { Pain intensity (day 1) } \\
\text { Child preoperative anxiety } \\
\text { Parent state anxiety } \\
\text { Parent trait anxiety }\end{array}$ & $\begin{array}{l}0.33^{*} \\
-0.13 \\
0.21 \\
-0.003\end{array}$ & $\begin{array}{l}0.11^{*} \\
0.02 \\
0.04\end{array}$ & $\begin{array}{l}0.12 \\
0.14 \\
0.17\end{array}$ \\
\hline Memory for pain-related fear (day 1) & $\begin{array}{l}1 \\
2 \\
3\end{array}$ & $\begin{array}{l}\text { Pain-related fear (day 1) } \\
\text { Child preoperative anxiety } \\
\text { Parent state anxiety } \\
\text { Parent trait anxiety }\end{array}$ & $\begin{array}{l}0.22 \\
-0.08 \\
0.11 \\
0.10\end{array}$ & $\begin{array}{l}0.05 \\
0.01 \\
0.02\end{array}$ & $\begin{array}{l}0.05 \\
0.05 \\
0.08\end{array}$ \\
\hline Memory for pain intensity (days 1-3) & $\begin{array}{l}1 \\
2 \\
3\end{array}$ & $\begin{array}{l}\text { Pain intensity (days } 1-3 \text { ) } \\
\text { Child preoperative anxiety } \\
\text { Parent state anxiety } \\
\text { Parent trait anxiety }\end{array}$ & $\begin{array}{l}0.30^{\star} \\
-0.07 \\
0.10 \\
0.02\end{array}$ & $\begin{array}{l}0.09^{*} \\
0.01 \\
0.01\end{array}$ & $\begin{array}{l}0.09 \\
0.09 \\
0.11\end{array}$ \\
\hline Memory for pain-related fear (days 1-3) & $\begin{array}{l}1 \\
2 \\
3\end{array}$ & $\begin{array}{l}\text { Pain-related fear (days 1-3) } \\
\text { Child preoperative anxiety } \\
\text { Parent state anxiety } \\
\text { Parent trait anxiety }\end{array}$ & $\begin{array}{l}0.46^{\star} \\
-0.05 \\
0.14 \\
0.22 \dagger\end{array}$ & $\begin{array}{l}0.21^{\star} \\
0.003 \\
0.07\end{array}$ & $\begin{array}{l}0.21 \\
0.22 \\
0.29\end{array}$ \\
\hline
\end{tabular}

${ }_{*} P<0.01$.

$+P<0.05$. 
postsurgical pain and fear during the recovery phase. Our findings revealed that parent, but not child, anxiety before surgery contributed to the development of negative biases in children's memories for pain-related fear 1 month later. Specifically, higher levels of parent trait anxiety led to children recalling higher levels of pain-related fear than they initially reported. Furthermore, children's initial ratings of postsurgical pain were significantly related to their subsequent pain memories. Children who initially reported higher levels of pain intensity and pain-related fear tended to recall higher levels pain intensity and pain-related fear. Moreover, we found that children who developed negatively biased memories of pain-related fear had higher postsurgical pain after surgery (ie, 3 days after discharge).

Parent, but not child, anxiety predicted the degree to which children developed negatively biased pain memories. A significantly higher proportion of 2- and 3-year-old children demonstrate preoperative distress behaviour compared with 4- to 10year-old children. ${ }^{8}$ With evolving cognitive and language abilities, children aged older than 4 years are better able to understand the context of their surgical and induction experiences. Given that fewer children aged older than 4 years exhibit anxiety around the time of induction, this could account for why a relation between child anxiety and pain memories was not found.

Our finding that parent trait anxiety was associated with children's memories for pain-related fear is in line with previous research with adolescents demonstrating that stable parent anxiety-related constructs (eg, parent catastrophizing) are robust predictors of adolescents' memories for postsurgical pain ${ }^{34}$ and long-term pain outcomes (eg, chronic postsurgical pain). ${ }^{36}$ Pain captures attention because of its inherent threat value. ${ }^{10}$ However, repeatedly emphasizing its threat value is maladaptive and may lead to the development of pain memory biases. Highly anxious parents may repeatedly attend to and reinforce their child's pain-related fear. Consequently, children may perceive their past surgery as more threatening, leading to more biased recall. This hypothesis is consistent with research demonstrating that parents of children aged 6 months to 15 years, who were high in catastrophic thinking, were more likely to be distressed and engage in pain-attending behaviour after a lumbar puncture. ${ }^{4}$

Another potential process underlying the influence of parent anxiety on children's memories for pain is the way in which parent-child dyads reminisce with one another about past painful experiences. ${ }^{31}$ Research has demonstrated the critical role of parent-child dyads reminiscing about negative emotional autobiographical events in influencing how children's memories of those events are framed, ${ }^{39}$ and this influence is especially strong for younger (vs older) children. ${ }^{2}$ Anxious parents may be more likely to use language that focuses on the pain-related and distressing details of the child's pain experience. Indeed, parents with high levels of anxious (ie, catastrophic) cognitions about child pain engaged in more pain-attending talk with their 6- to 9-yearold children after an experimental pain task. ${ }^{5}$ Therefore, parents with higher levels of trait anxiety may engage in less adaptive styles of reminiscing about pain with children, which could subsequently lead to negative biases in pain memories.

We found that parent trait anxiety predicted children's negatively biased memories for pain-related fear but not pain intensity. In the context of experimental and venipuncture pain, it was children's memories for the affective and not the sensory aspect of pain that were most susceptible to bias ${ }^{27,34}$ and the influence of parent anxiety. Among adolescents undergoing major surgery, parents' catastrophic thinking about pain (ie, their tendency to magnify the threat value of child pain) was predictive of negative biases in children's memories for the affective and not the sensory aspect of pain. ${ }^{34}$ Given that parental anxiety may cue threat perception and fear in children, it is not surprising that this fear-based, emotional construct would be most closely linked to the affective dimension of pain. Indeed, parental anxiety may influence the development of emotionally distressing pain memories through processes of fear learning.

The finding that parental anxiety was linked to memory of the average pain-related fear (over the first 3 days) vs the first day after surgery is consistent with past research on the link between parent catastrophizing about child pain and adolescent's memories of pain after major surgery. ${ }^{34}$ This could be explained by important differences between the pain experiences of children on day 1 vs several days after tonsillectomy. On day 1 after surgery, children are much more likely to take medication/ analgesics for their pain than on day 3. ${ }^{13}$ Thus, by day 3 after surgery, there are other ways that children must use to modulate or manage their pain, and so, parental influence may be greater.

Consistent with previous research across experimental, ${ }^{27}$ procedural, $^{6,30}$ and surgical ${ }^{34}$ pain, children who developed negatively biased pain memories had worse postsurgical pain in the recovery period. For the young children in this study, this was the case 3 days after surgery, pointing to the importance of assessing and managing children's postsurgical pain several days after discharge from hospital. Indeed, research has demonstrated that improving pain management is associated with a less biased recall of pain. In 8- to 11-year-old children undergoing injections, topical anesthetic and distraction were associated with less biased recall of pain (but not with less injection pain) as compared to children who received standard care (ie, no pain management). ${ }^{9}$

These findings should be viewed in light of limitations. We acknowledge, as we have in our past research with adolescents, ${ }^{34}$ that because children's ratings of pain intensity and painrelated fear were captured over several days by an average pain score, it is possible that their recall ratings may have underestimated the pain experienced. Moreover, pain intensity and pain-related fear on day 1 and their 3-day averages were intercorrelated. This correlation could be due to peak pain effects (ie, a tendency to remember the most intense pain). ${ }^{40}$ Similarly, several similar events (eg, postsurgical recovery days) may not be recalled as fundamentally different, ${ }^{38}$ which may further explain this intercorrelation. We further acknowledge that the SD for the 3-day average was smaller than that of day 1 ratings, which could have contributed to a lower error in the hierarchical regression models for the 3-day average ratings. Furthermore, we acknowledge that correlations between pain ratings were low, particularly between initial pain ratings and memory for pain as well as memory for pain intensity and memory for pain-related fear. However, this provides further support for the distinctiveness between sensory and affective aspects of pain memory. ${ }^{35}$ It is also in keeping with existing literature that has shown differential relationships between key variables and memory for affective vs sensory aspects of pain. ${ }^{32}$ This also provides support for the distinctiveness between initial and recalled pain ratings/ experience, which has been supported by past literature showing that children's pain recall ratings likely reflect memory for pain experience and not memory for pain ratings. ${ }^{45}$

In addition, children's observed anxiety was the sole assessment of child anxiety due to limitations of self-report of anxiety in this young age range. ${ }^{26}$ Other anxiety-related constructs were not assessed. Catastrophic thinking about pain has been found to be a robust predictor of pain memories in older children and adolescents. ${ }^{29,32,34}$ However, this construct has not been validated in, and may not be relevant for, younger children. ${ }^{11}$ Future research should examine 
other anxiety-related factors that may influence young children's memories for pain as well as observation of child anxiety at other time points (eg, after surgery upon waking).

In line with previous research, ${ }^{22}$ parents, as opposed to research staff, administered the FPS-R and the CFS to assess children's postsurgical pain and pain-related fear, which constitutes another limitation. Although more feasible and ecologically valid, parents' administration of scales may not be as reliable as those collected by researchers or clinicians. Moreover, with parents administering the scales, it is possible that children may have underreported their pain if they perceived their parents as highly anxious. Ideally, a research assistant would have collected these ratings. However, this may have been perceived as being too invasive for the families during the immediate postsurgical recovery period, which may have reduced retention in this longitudinal study. Future studies should consider the feasibility of phoning families each day to obtain postoperative pain ratings.

Finally, similar to previous research, ${ }^{32,44}$ the individual administering the pain scale at the time that pain was experienced was not the same individual who administered the same scale to assess children's recall of pain. Indeed, many researchers strive to have different individuals to administer the pain scale at the time of recall than the initial pain experience, so that children are potentially less likely to confound their memory of their ratings on the pain scale from their memory of the actual pain experience. Nevertheless, given limited empirical evidence to suggest that children's pain and recall ratings do not differ based on the individual administering the same scale, ${ }^{16}$ this methodological issue is worthy of further investigation.

In considering our findings, we note that the differences between mean pain and recall scores in this study were small. In terms of effect sizes, the partial correlation between parental anxiety and recalled fear, while controlling for initial fear, was in the moderate range $(r=0.25, P<0.05)$. This is similar to the moderate effect sizes found in previous research examining relationships between parental catastrophic thinking about child pain and adolescents' recall of postsurgical pain. ${ }^{34}$ Given that we did not examine future pain outcomes beyond memory in the current study, it is also important to situate the clinical significance of the current findings in the context of previous research linking memory biases of similar magnitude to real-world clinical outcomes, namely pain, fear, and distress at future pain experiences. Among healthy 8- to 12-year-old children undergoing experimental pain as well as adolescents undergoing major surgery (eg, spinal fusion and pectus repair), the effect sizes for the relationships between memory biases and future pain and fear were in the moderate to high range (ie, correlation coefficients within the range of $0.2-0.6) .{ }^{27,33}$ In more integrative and conservative models (ie, controlling for child age and sex, initial pain ratings, and anxiety), children's memories for experimental pain explained $15 \%$ of variance in future pain report. ${ }^{27}$ Finally, in adolescents undergoing major surgeries, the effect of children's memories for pain on future pain 4 months after surgery, at the time pain can transition to a chronic state, was $b=0.419$, and this was a medium effect size $(B=0.415) .{ }^{33}$

Beyond effect sizes, in terms of practical clinical significance of the effect, or whether it has a real genuine, noticeable effect on daily life, it is important to highlight that the main impetus for this study was based on clinical observations made by clinicians (ENT surgeons and anesthesiologists) who work with this population on a daily basis. They had long observed parental anxiety to be an issue in their clinical practice but were unsure of the potential outcomes of parental anxiety or the processes underlying this phenomenon. This study provides empirical evidence for a long-term consequence of parental anxiety by demonstrating the link to negative biases in children's memories for pain. We believe that demonstrating these effects lays the foundation for the development of clear and easily developed interventions to address parental anxiety. Nevertheless, given that the relationship between parental anxiety and children's memories for tonsillectomy pain has not been demonstrated in other studies, future research is needed to demonstrate the replicability of these findings and effects in the same clinical population. Furthermore, linking parental anxiety and children's memory biases to other real-world pain outcomes (eg, future procedural anxiety and pain) is needed to support the clinical significance of the results.

In conclusion, this study examined the influence of parent and child anxiety on the development of young children's postsurgical pain memory biases. Parent trait anxiety, but not child preoperative anxiety, significantly contributed to the development of young children's negatively biased memories for postsurgical pain. The development of pain-related fear and distress in youth is a major health concern as children who are afraid of procedurerelated pain may grow up to become adults who choose to avoid procedure-related pain (eg, vaccinations), putting both themselves and others at risk, and putting additional strain on the health care system. ${ }^{25}$ These findings underscore the need to target parent anxiety and to improve postsurgical pain management several days after surgery to alter young children's memories for pain-related fear and to potentially enhance children's pain trajectories.

\section{Conflict of interest statement}

The authors have no conflict of interest to declare.

\section{Acknowledgements}

This work was supported by the Society of Pediatric Psychology Targeted Research Grant, American Pain Society Future Leader in Pain Grant, Maternal Newborn Child, and Youth Strategic Clinical Network Health Outcomes Improvement Fund, and University of Calgary Faculty of Science Seed Grant was awarded to M. Noel. M. Pavlova was supported by the Alberta Strategy for Patient-Oriented Research Graduate Studentship. J. Vinall was supported by Louise \& Alan Edwards and Canadian Institutes of Health Research Fellowships.

\section{Article history:}

Received 1 June 2018

Received in revised form 29 November 2018

Accepted 17 December 2019

Available online 21 December 2018

\section{References}

[1] Bar-Haim Y, Lamy D, Pergamin L, Bakermans-Kranenburg MJ, van IMH. Threat-related attentional bias in anxious and nonanxious individuals: a meta-analytic study. Psychol Bull 2007;133:1-24.

[2] Bauer PJ, Larkina M, Deocampo J. Early memory development. In: Goswami U, editor. The handbook of childhood cognitive development. Hoboken: Wiley-Blackwell, 2010. p. 153-79.

[3] Beck AT, Clark DA. An information processing model of anxiety: automatic and strategic processes. Behav Res Ther 1997;35:49-58.

[4] Caes L, Vervoort T, Devos P, Verlooy J, Benoit Y, Goubert L. Parental distress and catastrophic thoughts about child pain: implications for parental protective behavior in the context of child leukemia-related medical procedures. Clin J Pain 2014;30:787-99. 
[5] Caes L, Vervoort T, Trost Z, Goubert L. Impact of parental catastrophizing and contextual threat on parents' emotional and behavioral responses to their child's pain. PAIN 2012;153:687-95.

[6] Chen E, Zeltzer LK, Craske MG, Katz ER. Children's memories for painful cancer treatment procedures: implications for distress. Child Dev 2000; 71:933-47.

[7] Chieng YJ, Chan WC, Klainin-Yobas P, He HG. Perioperative anxiety and postoperative pain in children and adolescents undergoing elective surgical procedures: a quantitative systematic review. J Adv Nurs 2014; 70:243-55.

[8] Chorney JM, Kain ZN. Behavioral analysis of children's response to induction of anesthesia. Anesth Analg 2009;109:1434-40.

[9] Cohen LL, Blount RL, Cohen RJ, Ball CM, McClellan CB, Bernard RS. Children's expectations and memories of acute distress: short- and longterm efficacy of pain management interventions. J Pediatr Psychol 2001; 26:367-74.

[10] Eccleston C, Crombez G. Pain demands attention: a cognitive-affective model of the interruptive function of pain. Psychol Bull 1999;125:356-66.

[11] Eccleston C, Fisher EA, Vervoort T, Crombez G. Worry and catastrophizing about pain in youth: a reappraisal. PAIN 2012;153: 1560-2.

[12] Fortier MA, Del Rosario AM, Rosenbaum A, Kain ZN. Beyond pain: predictors of postoperative maladaptive behavior change in children. Paediatr Anaesth 2010;20:445-53.

[13] Fortier MA, MacLaren JE, Martin SR, Perret-Karimi D, Kain ZN. Pediatric pain after ambulatory surgery: where's the medication? Pediatrics 2009; 124:e588-e595.

[14] Harris PA, Taylor R, Thielke R, Payne J, Gonzalez N, Conde JG. Research electronic data capture (REDCap) — a metadata-driven methodology and workflow process for providing translational research informatics support. J Biomed Inform 2009;42:377-81.

[15] Hicks CL, von Baeyer CL, Spafford PA, van Korlaar I, Goodenough B. The Faces Pain Scale-Revised: toward a common metric in pediatric pain measurement. PAIN 2001;93:173-83.

[16] Huq AH, Lindsay SJ, Roberts JF. Children's expectations and recollections of discomfort associated with dental treatment. Int $J$ Paediatr Dent 1992;2:11-16.

[17] Julian LJ. Measures of anxiety: State-Trait Anxiety Inventory (STAI), Beck Anxiety Inventory (BAI), and Hospital Anxiety and Depression ScaleAnxiety (HADS-A). Arthritis Care Res (Hoboken) 2011;63(suppl 11): S467-S472.

[18] Kain ZN, Caldwell-Andrews AA, Maranets I, McClain B, Gaal D, Mayes LC, Feng $\mathrm{R}$, Zhang $\mathrm{H}$. Preoperative anxiety and emergence delirium and postoperative maladaptive behaviors. Anesth Analg 2004;99:1648-54, table of contents.

[19] Kain ZN, Mayes LC, Caldwell-Andrews AA, Karas DE, McClain BC. Preoperative anxiety, postoperative pain, and behavioral recovery in young children undergoing surgery. Pediatrics 2006;118:651-8.

[20] Kain ZN, Mayes LC, Cicchetti DV, Caramico LA, Spieker M, Nygren MM, Rimar S. Measurement tool for preoperative anxiety in young children: the Yale Preoperative Anxiety Scale. Child Neuropsychol 1995;1:203-10.

[21] Lander J, Hodgins M, Fowler-Kerry S. Children's pain predictions and memories. Behav Res Ther 1992;30:117-24.

[22] MacLaren JE, Kain ZN. Prevalence and predictors of significant sleep disturbances in children undergoing ambulatory tonsillectomy and adenoidectomy. J Pediatr Psychol 2008;33:248-57.

[23] MacLaren JE, Thompson C, Weinberg M, Fortier MA, Morrison DE, Perret D, Kain ZN. Prediction of preoperative anxiety in children: who is most accurate? Anesth Analg 2009;108:1777-82.

[24] McMurtry CM, Noel M, Chambers CT, McGrath PJ. Children's fear during procedural pain: preliminary investigation of the Children's Fear Scale. Health Psychol 2011;30:780-8.
[25] McMurtry CM, Pillai Riddell R, Taddio A, Racine N, Asmundson GJ, Noel M, Chambers CT, Shah V, HELPinKids\&Adults Team. Far from "Just a Poke": common painful needle procedures and the development of needle fear. Clin J Pain 2015;31(10 suppl):S3-S11.

[26] Measelle JR, John OP, Ablow JC, Cowan PA, Cowan CP. Can children provide coherent, stable, and valid self-reports on the big five dimensions? A longitudinal study from ages 5 to 7. J Pers Soc Psychol 2005;89:90-106.

[27] Noel M, Chambers CT, McGrath PJ, Klein RM, Stewart SH. The influence of children's pain memories on subsequent pain experience. PAIN 2012; 153:1563-72.

[28] Noel M, Chambers CT, McGrath PJ, Klein RM, Stewart SH. The role of state anxiety in children's memories for pain. J Pediatr Psychol 2012;37: 567-79.

[29] Noel M, Chambers CT, Petter M, McGrath PJ, Klein RM, Stewart SH. Pain is not over when the needle ends: a review and preliminary model of acute pain memory development in childhood. Pain Manag 2012;2:487-97.

[30] Noel M, McMurtry CM, Chambers CT, McGrath PJ. Children's memory for painful procedures: the relationship of pain intensity, anxiety, and adult behaviors to subsequent recall. J Pediatr Psychol 2010;35:626-36.

[31] Noel M, Palermo TM, Chambers CT, Taddio A, Hermann C. Remembering the pain of childhood: applying a developmental perspective to the study of pain memories. PAIN 2015;156:31-4.

[32] Noel M, Pavlova M, McCallum L, Vinall J. Remembering the hurt of childhood: A psychological review and call for future research. Can Psychol 2017;58:58-68.

[33] Noel M, Rabbitts JA, Fales J, Chorney J, Palermo TM. The influence of pain memories on children's and adolescents' post-surgical pain experience: A longitudinal dyadic analysis. Health Psychol 2017;36: 987-95.

[34] Noel M, Rabbitts JA, Tai GG, Palermo TM. Remembering pain after surgery: a longitudinal examination of the role of pain catastrophizing in children's and parents' recall. PAIN 2015;156:800-8.

[35] Ornstein PA, Manning EL, Pelphrey KA. Children's memory for pain. J Dev Behav Pediatr 1999;20:262-77.

[36] Rabbitts JA, Fisher E, Rosenbloom BN, Palermo TM. Prevalence and predictors of chronic postsurgical pain in children: a systematic review and meta-analysis. J Pain 2017;18:605-14.

[37] Rocha EMT, von Baeyer CL. Anxiety influences children's memory for procedural pain. Pain Res Manag 2009;14:233-7.

[38] Rubin DC, Umanath S. Event memory: A theory of memory for laboratory, autobiographical, and fictional events. Psychol Rev 2015;122:1-23.

[39] Salmon K, Reese E. Talking (or not talking) about the past: the influence of parent-child conversation about negative experiences on children's memories. Appl Cogn Psych 2015;29:791-801.

[40] Schneider S, Stone AA, Schwartz JE, Broderick JE. Peak and end effects in patients' daily recall of pain and fatigue: a within-subjects analysis. J Pain 2011;12:228-35.

[41] Spielberger CD, Gorsuch RL, Lushene RE. State-Trait Anxiety Inventory. Palo Alto: Consulting Psychologists Press, 1970.

[42] Stinson JN, Kavanagh T, Yamada J, Gill N, Stevens B. Systematic review of the psychometric properties, interpretability and feasibility of self-report pain intensity measures for use in clinical trials in children and adolescents. PAIN 2006;125:143-57.

[43] Versloot J, Veerkamp JS, Hoogstraten J. Pain behaviour and distress in children during two sequential dental visits: comparing a computerised anaesthesia delivery system and a traditional syringe. Br Dent J 2008; 205:E2.

[44] von Baeyer CL, Marche TA, Rocha EM, Salmon K. Children's memory for pain: overview and implications for practice. J Pain 2004;5:241-9.

[45] Zonneveld LN, McGrath PJ, Reid GJ, Sorbi MJ. Accuracy of children's pain memories. PAIN 1997;71:297-302. 